\title{
Cure of Psoriasis and Arthritis when Addison's Disease Was Detected
}

\author{
Marcus Lind \\ Department of Medicine, NU Hospital Organization, Uddevalla, Sweden
}

\section{Key Words}

Psoriasis · Addison's disease · Cortisol · Arthritis

\begin{abstract}
Introduction: Corticoid therapy is well-known to improve the symptoms of psoriasis. Addison's disease is an autoimmune disease which leads to a loss of cortisol production in the adrenal glands. This case report describes a patient with wide-spread psoriasis for 34 years who was cured when Addison's disease was detected and substitution to reach normal biological cortisol levels was introduced.

Case Report: A 59-year-old man was diagnosed with Addison's disease. He had been tired for several years and had had difficulties in continuing his work. His brother had Addison's disease and recommended him to make a screen for the disease. Synacthen test diagnosed Addison's disease with a clear deficiency of cortisol production. After substitution with hydrocortisone the patient's constitution improved rapidly and he felt no longer tired during work. At the same time, all skin lesions of psoriasis disappeared as well as aches in several joints, both symptoms having been present for a couple of decades. Previously, salves of cortisol had been used to reduce the symptoms of psoriasis, but now, 1-2 years later, after the treatment of Addison's disease, no symptoms in the skin or joints have reoccurred.

Conclusions: This report illustrates that Addison's disease, although a rare condition, should be kept in mind before treatment of psoriasis is started. Especially if other symptoms such as fatigue are present, a screening test of serum cortisol in the morning should be liberally made. The report also illustrates a need of examining corticoid levels in patients with psoriasis compared to the general population.
\end{abstract}

\section{Introduction}

Psoriasis is a common disease with commonly wide-spread skin lesions [1]. Corticoid therapy is well-known to improve the symptoms of psoriasis. Addison's disease is an autoimmune disease which leads to a loss of cortisol production in the adrenal glands [2]. 
Addison's disease is often accompanied by tiredness and a disturbed salt regulation due to a deficiency of the hormones aldosterone and cortisol normally produced in the adrenal glands [2]. In the acute phase of Addison's disease stomach aches and nausea also exist.

Since cortisol levels are important for the symptoms of psoriasis, the question arises whether patients with suboptimal cortisol production develop psoriasis more easily. This case report describes a patient with wide-spread psoriasis for 34 years, which was cured when Addison's disease was detected and substitution to reach normal biological cortisol levels was introduced.

\section{Case Report}

At 59 years of age, this patient was diagnosed with Addison's disease in our endocrinologic outpatient clinic. He had a brother with Addison's disease who recommended him to screen for the disease since the present patient had had similar symptoms of grave fatigue for several years. Serum cortisol showed a low morning value when tested in the primary care unit and Addison's disease was confirmed by synacthen test in our clinic.

When the patient was 28 years old, psoriasis appeared on the skull, arms, elbows, knees, hollows of the knees, calves and hands. Since then, he has been using corticoid salves to reduce these symptoms. He has also had aches in several joints, although no diagnosis has been made and no specific treatment has been prescribed. Both proximal and distal joints of the fingers, the hip and the distal joint of the big toe have been affected.

After diagnosis of Addison's disease I prescribed hydrocortisone $20 \mathrm{mg}, 1$ pill in the morning and half a pill in the evening, to keep normal biological cortisol levels. The fatigue, which had been present for many years, rapidly disappeared and the patient had no longer any limitations in managing his work, which had previously been a problem. Simultaneously, the skin lesions of psoriasis, which had been present for 30 years, totally disappeared. There was no longer any need for corticoid salves. Furthermore, the aches in all joints disappeared.

\section{Discussion}

This case report illustrates two important aspects to consider in the clinical treatment of psoriasis and arthritis as well as in research concerning these conditions. It illustrates that Addison's disease, although a rare condition, should be kept in mind before treatment of psoriasis or arthritis is started. Especially if other symptoms such as fatigue are present, a screening test of serum cortisol in the morning should be made. The report also illustrates a need of examining corticoid levels in patients with psoriasis compared to the general population. It could be questioned whether psoriasis or arthritis develops in some patients due to a suppressed cortisol production in the adrenal glands.

In the present report, a patient had skin lesions of psoriasis over 3 decades, which disappeared when Addison's disease was detected and substitution to reach normal cortisol levels was introduced. It is difficult to know for how long the patient had had a reduced levels of cortisol and whether this could be the cause of psoriasis during the whole course of the disease. The levels of cortisol had certainly been substantially reduced for several years, since the patient could remember the grave fatigue he had felt for many years, a fatigue which rapidly disappeared when Addison's disease was detected and treated. Hence, it is also possible that a slight suppression even at an early stage of psoriasis had existed.

It is possible that a substitution dose of hydrocortisone could cause a slight increase in the cortisol levels compared with normal levels, thus influencing the treatment of 
psoriasis. However, the cortisol levels obtained by hydrocortisone could lead to an increased risk of osteoporosis, and doses of 15-25 mg of hydrocortisone have been found to be more appropriate [3]. Hence, in the present case, the finding either indicates that suppressed levels of cortisol can lead to the development of psoriasis or that a small dose of hydrocortisone could be a potent therapy in patients with psoriasis.

Psoriasis has been described as one of the most typical psychocutaneous diseases [4]. The hypothalamic-pituitary-adrenal (HPA) axis function has been suggested as essential in the development of psoriasis and several psychiatric disorders and psychosomatic diseases. However, the evidence of a reduced response of the HPA axis in psoriasis after psychosocial stress is diverging and difficult to study [4]. In psoriasis arthritis, TNF- $\alpha-$ antibodies, which have a potent effect on symptoms, have proved to increase the relative production of cortisol in the adrenal glands compared with other adrenal hormones [5]. This could indicate the importance of a balanced cortisol production for both psoriasis and psoriasis arthritis. This report supports the importance of an adequate endogenous cortisol balance in the pathogenesis and treatment of psoriasis and psoriasis arthritis. 


\section{References}

1 Stankier L: Diseases of the skin. Psoriasis. Br Med J 1974;1:27-29.

-2 Nieman LK, Chanco Turner ML: Addison's disease. Clin Dermatol 2006;24:276280.

-3 Løvås K, Gjesdal CG, Christensen M, Wolff AB, Almås B, Svartberg J, Fougner KJ, Syversen U, Bollerslev J, Falch JA, Hunt PJ, Chatterjee VK, Husebye ES: Glucocorticoid replacement therapy and pharmacogenetics in Addison's disease: effects on bone. Eur J Endocrinol 2009;160:993-1002.

4 Karanikas E, Harsoulis F, Giouzepas I, Griveas I: Stimulation of the hypothalamic-pituitary-adrenal axis with corticotropin releasing hormone in patients with psoriasis. Hormones (Athens) 2007;6:314-320.

5 Atzeni F, Sarzi-Puttini P, DePortu S, Cutolo M, Carrabba M, Straub RH: In etanercept-treated psoriatic arthritis patients clinical improvement correlated with an increase of serum cortisol relative to other adrenal hormones. Clin Exp Rheumatol 2008;26:103-108. 